Methods: We analyzed data based on the preliminary results of the patients registry from the ongoing project "Patient's Opinion on Covid-19 in Rheumatic Diseases (POPCORD)". The registry contains data collected directly from Russian-speaking patients with different RD who completed a special questionnaire, located on the website https://revmo-covid.ru/. The questionnaire includes information on whether the patient had COVID-19 and related information, as well as information on rheumatic disease and its treatment.

Results: 1050 patients (92\% from Russian Federation, 8\% from other locations) completed the survey - 93\% females, mean age 40,28 $\pm 11,26(\mathrm{M} \pm \mathrm{SD}$, median 38 [32;48]), BMI 24,52 $\pm 5,38$ (median 23,42 [21;27]), disease duration 7,91 $\pm 8,46$ (median 5,00 [2;11]). Diagnoses reported: rheumatoid arthritis (49\%), ankylosing spondylitis (14\%), systemic lupus erythematosus (13\%), psoriatic arthritis $(6 \%)$, Sjogren's disease $(5 \%)$, systemic sclerosis $(3 \%)$, other $(10 \%) .51 \%$ of respondents indicated concomitant conditions: arterial hypertension $(19 \%)$, obesity (15\%), kidney disease (11\%), cardiovascular disease (7\%), liver disease $(6 \%)$, IBD $(5 \%)$, other lung diseases, including ILD (5\%). $96 \%$ of patients reported constant intake of antirheumatic drugs: steroids (37\%), NSAIDs (46\%), hydroxychloroquine (22\%), csDMARDs (66\%), bDMARDs (17\%), tsDMARDs (2\%). 344 $(31,8 \%)$ patients reported COVID-19 or suspected SASR-CoV2 viral pneumonia, $282(84,4 \%)$ were treated as outpatients, $52(15,6 \%)$ were hospitalized. Relatives or physicians reported 3 cases of death related to COVID-19 $(0,9 \%$ of total mortality, 5,8\% of in-hospital mortality). Table 1 contains odds ratios for the main factors, related to hospitalization, based on the results of the survey. Analysis of the survey participants' parameters (patients' characteristics, treatments etc.) did not reveal any significant variables, related to increased risk of hospitalization. No increased risk related to any groups of anti-rheumatic drugs or any particular medications was found either. Risk of hospitalization was significantly lower in patients with ankylosing spondylitis.

Table 1. Factors, related to hospitalization due to COVID-19 in patients with RD.

\begin{tabular}{|c|c|c|}
\hline Factor & OR $[95 \% \mathrm{Cl}]$ & $p$ \\
\hline Age $\geq 50$ y.o. & $2,12[1,14 ; 3,94]$ & 0,99 \\
\hline Age $\geq 65$ y.o. & $9,04[2,10 ; 38,98]$ & 1,00 \\
\hline Female & $1,73[0,70 ; 4,25]$ & 0,92 \\
\hline Body mass index $\geq 35$ & $2,09[0,71 ; 6,10]$ & 0,88 \\
\hline Current smoking & $0,47[0,11 ; 2,06]$ & 0,24 \\
\hline Smoking in the past & $1,43[0,83 ; 2,48]$ & 0,92 \\
\hline Diagnosis of ankylosing spondylitis & $0,25[0,06 ; 1,05]$ & 0,02 \\
\hline \multicolumn{3}{|l|}{ Comorbid conditions: } \\
\hline - Diabetes mellitus & $3,39[1,07 ; 10,77]$ & 0,99 \\
\hline - Cardiovascular disease & $1,91[0,72 ; 5,08]$ & 0,94 \\
\hline - Asthma & $5,42[1,07 ; 27,61]$ & 0,99 \\
\hline - COPD & $0,42[0,05 ; 3,26]$ & 0,34 \\
\hline - Kidney disease & $1,57[0,71 ; 3,50]$ & 0,91 \\
\hline - Liver disease & $0,90[0,26 ; 3,18]$ & 0,57 \\
\hline - Neurological disorders & $5,42[0,75 ; 39,36]$ & 0,99 \\
\hline - No concomitant conditions & $0,70[0,40 ; 1,25]$ & 0,14 \\
\hline \multicolumn{3}{|l|}{ Therapy: } \\
\hline - Oral steroids & $1,37[0,82 ; 2,29]$ & 0,91 \\
\hline - NSAIDs & $1,12[0,67 ; 1,87]$ & 0,72 \\
\hline - Traditional DMARDs & $1,39[0,89 ; 2,17]$ & 0,94 \\
\hline - Biologic DMARDs & $1,15[0,55 ; 2,43]$ & 0,72 \\
\hline $\begin{array}{l}\text { Targeted synthetic DMARDs (JAK-inhibitors, apremilast) } \\
\text { Level of concern about COVID-19: }\end{array}$ & $3,25[0,75 ; 14,03]$ & 0,97 \\
\hline - Low level / no concern about COVID-19 & $0,57[0,28 ; 1,17]$ & 0,08 \\
\hline - Medium level of concern & $0,60[0,28 ; 1,28]$ & 0,12 \\
\hline - High level of concern & $1,69[1,03 ; 2,76]$ & 0,99 \\
\hline
\end{tabular}

Conclusion: Survey of patients with RD did not show significantly increased risk for hospitalization due to COVID-19 in relation with specific diagnosis, any anti-rheumatic medications, as well as comorbid conditions and main patient characteristics. Diagnosis of ankylosing spondylitis related to significantly lower risk of hospitalization.

Acknowledgements: Dr Anna S Misiyuk from V.A. Nasonova Institute of Rheumatology, Moscow, Russia.

The COVID-19 Russian patient experience survey is conducted on behalf of Russian Rheumatology Association "Nadezhda". The project was partially granted by BIOCAD.

Disclosure of Interests: None declared

DOI: 10.1136/annrheumdis-2021-eular.4065

\section{POS1267 CLINICAL COURSE OF NEW SARS-COV-2 INFECTION IN CHILDREN WITH RHEUMATIC DISEASES: RETROSPECTIVE STUDY OF SINGLE PEDIATRIC RHEUMATOLOGY CENTER}

I. Nikishina ${ }^{1}$, Z. Kolkhidova ${ }^{1}$, V. Matkava ${ }^{1}$, A. Shapovalenko ${ }^{1}$, S. Arsenyeva ${ }^{1}$, M. Kaleda', E. Fedorov', S. Salugina ${ }^{1} .{ }^{1}$ V.A. Nasonova Research Institute of Rheumatology, Pediatric Department, Moscow, Russian Federation
Background: About one year ago at the beginning of the new SARS-CoV-2 infection pandemic, it was expected, that rheumatic disease (RD) and immunosuppressive therapy could predispose to a more severe course of the Covid-19 infection. At that time, it was unspeakable how this infection would occur in children with RB in compare to adults. Now it's time to draw preliminary conclusions. Objectives: to analyze all features of COVID19 infection in children with RD Methods: The retrospective single center study of all patients with pediatric RD who had the COVID19 infection up to the end of January 2021. It was analyzed all variety of clinical manifestations, changing in therapy at the time of COVID-19 and after it, the dynamics of the RD and consequences of the new viral disease. Results: The study included 66 patients under the age of 18 with various RS, including autoinflammatory disease (AIDs). The diagnostic distribution, sex ratio age and previous therapy present in the Table 1. There were patients treated with DMARDS (methotrexate -47 , hydroxychloroquine -3 , mycophenolate mofetil -2 colchicine -1), NSAID monotherapy -6 , systemic steroids $-10 ; 29$ patients recived Biologics, including combination with methotrexate. None of our patients had a severe course of the COVID-19, none of 66 patients was hospitalized for emergency indications in the intensive care unit or in other specialized departments. The diagnosis of Covid-19 infection was based on different evidences: positive PCR in $15(23 \%)$ cases, high levels of immunoglobulins G and M - in 64 (97\%) and $7(11 \%)$ respectively, viral pneumonia was confirmed by CT in $2(10 \%)$ cases. A manifest clinical picture presented just in 21 (32\%) of patients, the others were carried the disease asymptomatically. Among the clinical manifestations of SARS-CoV-2 that were observed in our patients were the following: fever, as the most frequent symptom - $19(90 \%)$, rhinitis - $12(57 \%)$, anosmia - $10(48 \%)$ sore throat - $3(14 \%)$, arthralgia/myalgia in $4(19 \%)$, rash - $1(5 \%)$ case, cough - $4(19 \%)$ cases. it is important to note that until July 2020 , only 4 patients were registered, and in the next 6 months -62 . The treatment against Covid-19 in our patients with clinical symptoms included: NSAID monotherapy $-4(19 \%)$, Systemic steroids -2 (10\%), Hydroxychloroquine $-2(10 \%)$, Antibiotics-5 (24\%).

Table 1. Clinical characteristics of children with RD according to presence of symptoms of Covid-19

\begin{tabular}{|c|c|c|c|c|}
\hline \multicolumn{2}{|l|}{ Parameter } & $\begin{array}{c}\text { Covid-19 } \\
\text { symptomatic } \\
\text { course }(n=21)\end{array}$ & $\begin{array}{l}\text { Covid-19 asymp- } \\
\text { tomatic course } \\
\qquad(n=45)\end{array}$ & $\begin{array}{c}\mathrm{p}- \\
\text { values }\end{array}$ \\
\hline \multicolumn{2}{|l|}{ Sex ratio $(F / M)$} & $9 / 12$ & $30 / 15$ & ns \\
\hline \multicolumn{2}{|c|}{ Duration of RD (Me. Years) } & $4(2 ; 6)$ & $4(2 ; 7)$ & ns \\
\hline Age & $\mathrm{Me}$ & $15(13 ; 17)$ & $11(8 ; 13.5)$ & $<0,001$ \\
\hline \multirow[t]{2}{*}{ (years) } & $<10$ yrs & 1 & 19 & $<0,05$ \\
\hline & $>10$ yrs & 20 & 26 & $<0,001$ \\
\hline \multicolumn{2}{|c|}{ Covid-19 evidences IgG Covid-19 + } & 19 & 45 & ns \\
\hline & IgM Covid-19+ & 3 & 4 & ns \\
\hline & PCR Covid-19+ & 11 & 0 & ns \\
\hline \multirow[t]{6}{*}{ Diagnosis of RD } & JIA non-systemic & 14 & 31 & $\mathrm{~ns}$ \\
\hline & SJIA & 1 & 3 & ns \\
\hline & SLE & 4 & 1 & ns \\
\hline & JDM & 1 & 1 & ns \\
\hline & Scleroderma & & 6 & ns \\
\hline & AIDs (CAPS, FMF, FOP) & 1 & 3 & ns \\
\hline \multirow[t]{7}{*}{ Previous therapy } & NSAID monotherapy & 3 & 6 & $\mathrm{~ns}$ \\
\hline & DMARDs & 16 & 33 & ns \\
\hline & Systemic steroids & 4 & 7 & ns \\
\hline & TNF-inhibitors & 5 & 12 & ns \\
\hline & Abatacept & 1 & 3 & ns \\
\hline & $\begin{array}{l}\text { IL6-inhibitors (Tocilizumab, } \\
\text { Sarilumab) }\end{array}$ & 0 & 2 & ns \\
\hline & JAK-kinase inhibitors & 1 & 2 & ns \\
\hline \multicolumn{2}{|c|}{ Worsening/flare of RD after Covid-19 infection } & 5 & 0 & $p<0,05$ \\
\hline
\end{tabular}

Conclusion: Our study suggests that the presence of RD and immunosuppression therapy does not lead to an increased frequency or severe course of Covid-19 infection in children. The final conclusion will be drawn as the data accumulates.

Disclosure of Interests: None declared

DOI: 10.1136/annrheumdis-2021-eular.4101

\section{POS1268 \\ COVID-19 VACCINE HESITANCY AMONG RHEUMATOLOGY PATIENTS RECEIVING INFLUENZA VACCINE}

V. Valerio ${ }^{1}$, H. C. Shen ${ }^{2}$, E. Field ${ }^{1}$, E. G. Mcdonald ${ }^{3}$, A. Turner ${ }^{4}$, S. Bernatsky ${ }^{3}$, M. Hudson ${ }^{5}$, I. Colmegna ${ }^{3} .^{1}$ The Research Institute of the McGill University Health Care Centre, Medicine, Montreal, Canada; ${ }^{2}$ University of Montreal, Medicine, Montreal, Canada; ${ }^{3}$ Mc Gill University Health Care Centre, Medicine, Montreal, Canada; ${ }^{4}$ Mc Gill University Health Care Centre, Nursing, Montreal, Canada; ${ }^{5}$ Jewish General Hospital, Medicine, Montreal, Canada 
Background: Adults with rheumatic diseases are a COVID-19 vulnerable population with potential increased risk for severe infection. COVID-19 vaccines are a key strategy to ending the pandemic. Unfortunately, fears about vaccines, some of which are propagated by misinformation, are common and may prevent or inappropriately delay vaccination. Refusal or uncertainty to get a vaccine despite its availability is known as vaccine hesitancy.

Objectives: This study aims at defining causes of COVID-19 vaccine hesitancy among rheumatology patients.

Methods: Between November and December 2020, a cross-sectional survey was completed by rheumatology patients presenting to a large Canadian tertiary-care center for influenza immunization. COVID-19 risk factors, previous COVID-19 infection, the likelihood of getting a future COVID-19 vaccine (scale 0-10), and contextual, individual, and vaccine-specific potential determinants of vaccine hesitancy were assessed. Patients were classified into 5 groups based on how likely they were to get a future COVID-19 vaccine $(0=$ not likely at all; $2.5=$ unlikely; $5=$ intermediate; $7.5=$ likely; $10=$ highly likely). A machine learning approach (XgBoost) was used to fit univariate models for a multi-class correlation. Results: 157 rheumatology patients completed the survey. Most were females $(n=112,71 \%)$ with a mean age of 54.6 (standard deviation 17.9). The majority ( $73 \%$ ) had tertiary-education, and $46 \%$ were employed at the time of the survey. The most common rheumatology diagnoses were rheumatoid arthritis $(n=90$, $58 \%)$, systemic lupus erythematosus/vasculitis $(n=41,26 \%)$ and spondyloarthropathies $(n=39,25 \%)$. Most patients were on immunosuppressors ( $n=93$, $59 \%)$. Only half ( $n=85,54 \%)$ were highly likely to accept a future COVID vaccine, $17 \%(n=26)$ likely, $19 \%(n=30)$ intermediate, $6 \%(n=10)$ unlikely, and $4 \%$ $(n=6)$ not likely at all. One hundred thirty-five patients $(86 \%)$ previously received the flu vaccine, whereas $6 \%(n=10)$ previously rejected it. Only three patients were previously diagnosed with COVID-19 (2\%) one of whom was hospitalized. Eighty-seven patients (56\%) considered that the COVID-19 vaccine should be mandatory, and $101(65 \%)$ that they should receive it. Most respondents were somewhat concerned about receiving a future COVID-19 vaccine $(n=116,76 \%)$ despite that $65 \%$ believed that vaccines benefits outweighed their risks. Almost all, $(n=145,96 \%)$ believed that governmental decisions about vaccines are in the best interest of the population, while less than half $(n=70,45 \%)$ were confident that pharmaceutical companies would provide safe and effective vaccines. One hundred participants $(65 \%)$ denied feeling social pressure to get the vaccine, and $55 \%(n=81)$ were willing to pay for the vaccine. Feeling social pressure about getting a COVID-19 vaccine, having severe concerns about receiving a future COVID-19 vaccine, distrust in pharmaceutical companies, lower education, and doubts of whether vaccines benefits outweigh their risks, were negatively associated with COVID vaccine acceptance.

Conclusion: Forty six percent of rheumatology patients being immunized against influenza showed at least some hesitancy towards COVID-19 vaccination. Multiple contextual, individual, and vaccine-related factors may contribute. Targeted educational strategies, including producing and communicating data on vaccine safety, may help promote vaccine uptake in this potentially vulnerable population.

Disclosure of Interests: None declared

DOI: 10.1136/annrheumdis-2021-eular.4150

\section{Spine, mechanical musculoskeletal problems, local soft tissue disorders}

\section{POS1269 THE UTILITY OF ULTRASOUND TO PREDICT PATIENT OUTCOMES IN SHOULDER PAIN: A PROSPECTIVE OBSERVATIONAL STUDY OF 500 PATIENTS}

${\text { G. } \operatorname{Tran}^{1,2,3}, \text { S. Kingsbury }}^{1,2}$, E. Hensor ${ }^{1,2}$, P. G. Conaghan ${ }^{1,2}{ }^{1}$ Leeds Institute of Rheumatic and Musculoskeletal Medicine, Chapel Allerton Hospital, Leeds, United Kingdom; ${ }^{2}$ National Institute for Health Research Leeds Biomedical Research Centre, Chapel Allerton Hospital, Leeds, United Kingdom; ${ }^{3}$ Harrogate and District NHS Foundation Trust, Department of Rheumatology, Harrogate, United Kingdom

Background: Shoulder pain is common and persistent, with a large socioeconomic burden. Ultrasound (US) scans are used for diagnosing and managing shoulder pain, but the extent to which it informs management and improves outcomes is unknown. A recent retrospective study identified groups with different patterns of US pathologies.

Objectives: To confirm the existence of US-based groups of shoulder pain and determine if US-detected pathology (grouped or individual) predicts outcomes in the context of usual care. Response to local steroid injection was also evaluated. Methods: This was a 6 month, prospective, single centred, community based, observational cohort study. Inclusion: shoulder pain, $\geq 18$ years, first shoulder US. Exclusion: shoulder surgery, inflammatory arthritis, steroid/physiotherapy in prior 6 weeks. Standardised reporting for 10 US pathologies was employed. Latent class analysis (LCA) identified pathology-based groups. Multiple linear regression analysis explored associations between baseline pathologies, subsequent treatment and 6-month Shoulder Pain and Disability Index (SPADI). Growth mixture modelling (GMM) identified groups with common trajectories of change. Results: Of 500 patients (mean age 53.6; 52\% female), 330 completed follow-up. LCA identified 4 groups: bursitis without acromioclavicular joint degeneration (ACJD) (group 1), bursitis with ACJD (group 2), rotator cuff tear (group 3), no bursitis/tear (group 4). SPADI was higher at baseline for tears (55.1 vs. 49.7-51.3) (overall $p=0.005)$, but groups did not differ at 6 months $(p=0.379)$ (Table 1). No individual pathologies predicted 6-month outcomes. Response to baseline injection at week 2 did not differ between groups ( $p=0.423)$. GMM found 4 trajectories; the majority of patients followed trajectory 1 (little change), irrespective of US pathology group $(79 \%, 77 \%, 87 \%, 70 \%$ of US groups $1-4$ respectively) (Figure 1 ). Conclusion: This is the largest prospective study involving US of symptomatic shoulders, and the first to investigate groups with distinct patterns of US pathologies in predicting outcome. US-based classification of pathology (as groups or individually) did not predict 6-month outcomes with current treatments and there were no differences in short-term response to steroids between groups. The role of routine diagnostic US for shoulder pain needs consideration; it may be useful if evidence-based therapies for specific pathologies are established.

\section{Table 1. Predictors of SPADI score at 6 months}

Baseline characteristic

Coefficient $^{*}(95 \% \mathrm{Cl})$, p-value

Pathology group:Bursitis w/o ACJ degeneration (group 1)

Bursitis with ACJ degeneration (group 2)

$\mathrm{RC}$ tear (group 3 )

No bursitis, no RC tear (group 4)

Injection at scan

Age, years

Female

Symptom duration, months

Uses arms to rise from chair

Physiotherapy before baseline

1 injection before baseline

$\geq 2$ injections before baseline

Total SPADI

Shoulder activity score

P-SEQ score

Brief IPQ score

HADS score

Constant ${ }^{\star \star}$ Reference $.08(-5.15,5.32), p=0.975$ $5.01(-1.48,11.50), p=0.130$ $1.98(-4.00,7.96), p=0.516$ $-0.01(-0.17,0.15), p=0.898$ $-1.51(-5.42,2.40), p=0.448$ $0.00(-0.04,0.05), p=0.879$ $2.65(-1.50,6.81), p=0.210$ $-0.23(-4.78,4.31), p=0.920$ $2.35(-2.87,7.56), p=0.377$ $6.53(-2.50,15.57), p=0.156$ $0.62(0.35,0.89), p<0.001$ $-0.59(-1.15,-0.04), p=0.037$ $-0.20(-0.46,0.07), p=0.141$ $0.19(-0.16,0.54), \mathrm{p}=0.298$ $0.01(-0.38,0.41), p=0.947$ $35.44(29.50,41.38), p<0.001$

*Interpreted as unit difference in Rasch-transformed SPADI score per 1 additional unit of the independent variable ${ }^{\star \star}$ Estimated SPADI at 26 weeks in patients in the reference category for all categorical variables and with mean values for continuous covariates. ACJ=acromioclavicular joint; HADS=hospital anxiety and depression scale; IPQ=illness perception questionnaire $\mathrm{P}$-SEQ=pain self-efficacy questionnaire; $\mathrm{RC}=$ rotator cuff; $\mathrm{SPADI}=$ shoulder pain and disability index

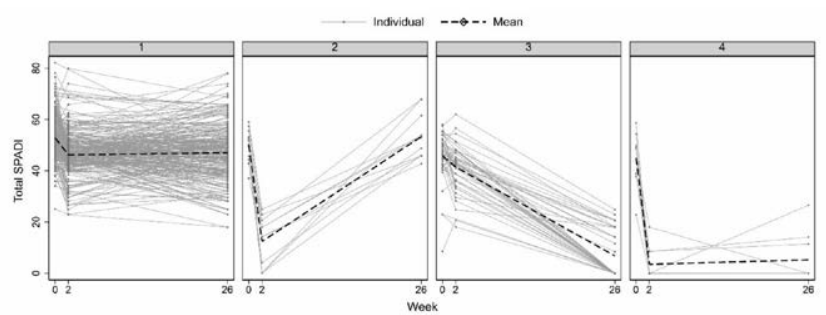

Figure 1. Trajectories of total SPADI over time by the pathology groups found and response to injections

Acknowledgements: This research was funded by the National Institute for Health Research (NIHR) Leeds Biomedical Research Centre (BRC) and by a NIHR Doctoral Research Fellowship (GT; DRF-2016-09-159). The views expressed are those of the authors and not necessarily those of the NIHR or the Department of Health and Social Care. This study was also part-funded through the Arthritis Research UK Leeds Experimental Osteoarthritis Treatment Centre (20083).

Disclosure of Interests: None declared

DOI: 10.1136/annrheumdis-2021-eular.292

\section{POS1270 OF 234 INFLAMMATORY BOWEL DISEASE PATIENTS}

B. M. Fernandes ${ }^{1}$, M. Bernardes ${ }^{1}$, D. R. Gonçalves ${ }^{2}$, F. Magro ${ }^{3}$, L. Costa ${ }^{1}$

${ }^{1}$ Centro Hospitalar Universitário São João, Rheumatology, Porto, Portugal; 